\title{
On the importance of precision medicine for neuropathic pain
}

\section{Introduction}

At the recent October 2018 EFIC congress in Bergamo (Italy), discussing 'Unmet Needs in Neuropathic Pain', in the presence of many neuropathic pain specialists, Professor Nadine Attal pointed out that most neuropathic pain (NP) patients prefer non-drug therapy, and that drugs recommended may be poorly effective. The fact that most patients prefer non-drug therapy is already a clear signal, that we are not proceeding according to patient-centered lines. She also highlighted that drugs accepted in the general guidelines perform poor and in time ever poorer, with the Numbers Needed to Treat (NNT) according to the last meta-analysis of pregabalin being $7 !^{1}$ This means that 7 patients have to be treated to achieve in 1 patient at least $50 \%$ pain reduction. She presented data quite worrying, the presence of the relation between trial methodology and outcome: the more rigorous study designs, the smaller the efficacy of drugs evaluated. That is why the initial NNT of pregabalin is circa 4, rose to 7 in the most recent meta-analysis. This of course is also related to the fact that the bias against publishing negative studies is decreasing in time. Another speaker, Dr. M Hannah from the UK pointed out that more than $75 \%$ of NP patients do not respond to treatment, that we therefore need a new and different paradigm of treatment. This approach might probably be based on molecules with broad acting mechanisms of action (MOA), to get a better grip on non-responders, and moreover, we should think laterally in designing new tools to evaluate neuropathic pain.

\section{Rise of precision pain medicine}

So clearly there is a core problem in the treatment of peripheral neuropathic pain, amongst pain in diabetic neuropathy (PDN), chronic idiopathic axonal polyneuropathy (CIAP), chemotherapyinduced peripheral neuropathy (CIPN), post-herpetic neuralgia (PHN) and thin-fiber neuropathy (SFN). This core problem is that we do not exactly know which pathogenesis plays a relevant role in which disorder. Pain in all these variants is generally treated in the same way with gabapentin, pregabalin, amitriptyline, duloxetine or patches based on lidocaine and capsaicin. This results in disappointing high NNT's, as we depicted in Table 1. However, every condition will have its own pathogenesis. Even within a category, for example PHN, three sub-variants are already distinguished on the basis of different pathogenesis. ${ }^{6}$ In practice, this means a so called 'ex juvantibus' treatment: first we try one and then the other co-analgesic, without much if any insight why; just try and see. The possibility to arrive at an individualized treatment, in which pathogenesis and MOA of the analgesic are adapted to each other, seems still future music. In 2010, we started together with a pharmacist, the development and evaluation of a number of compounded creams based on, ketamine, amitriptyline, clonidine, baclofen, gabapentin, loperamide and phenytoin. We deliberately opted for high concentrations, for example for amitriptyline we selected $5 \%$ to $10 \%$, for ketamine $10 \%$ and for phenytoin $10 \%$ to $20 \%$. In the field of topical analgesics, formal doseresponse studies have never been done. ${ }^{7}$ Nicholas J Schork ${ }^{8}$ published a comment in Nature, 'Personalized medicine: Time for one-person trials', and he pointed out that precision medicine requires a different
Volume 6 Issue 6 - 2018

\author{
Jan M Keppel Hesselink, David J Kopsky \\ Institute for Neuropathic Pain, Netherlands
}

Correspondence: David J Kopsky, Institute for Neuropathic Pain,Vespuccistraat 64-III, I056 SN Amsterdam, Netherlands, Emailimfo@neuropathie.nu

Received: October 25, 20I8 | Published: November 16, 2018

type of clinical trial that focuses on individual, not average, responses to therapy. He raised awareness to the importance of N-of-1 trials, which will be a crucial part of a new treatment paradigm.

Table I Numbers needed to treat for different neuropathic pain analgesics, from different sources. The table is not meant to compare, only to demonstrate that all current frequently used analgesics in neuropathic pain are only effective in a few patients.

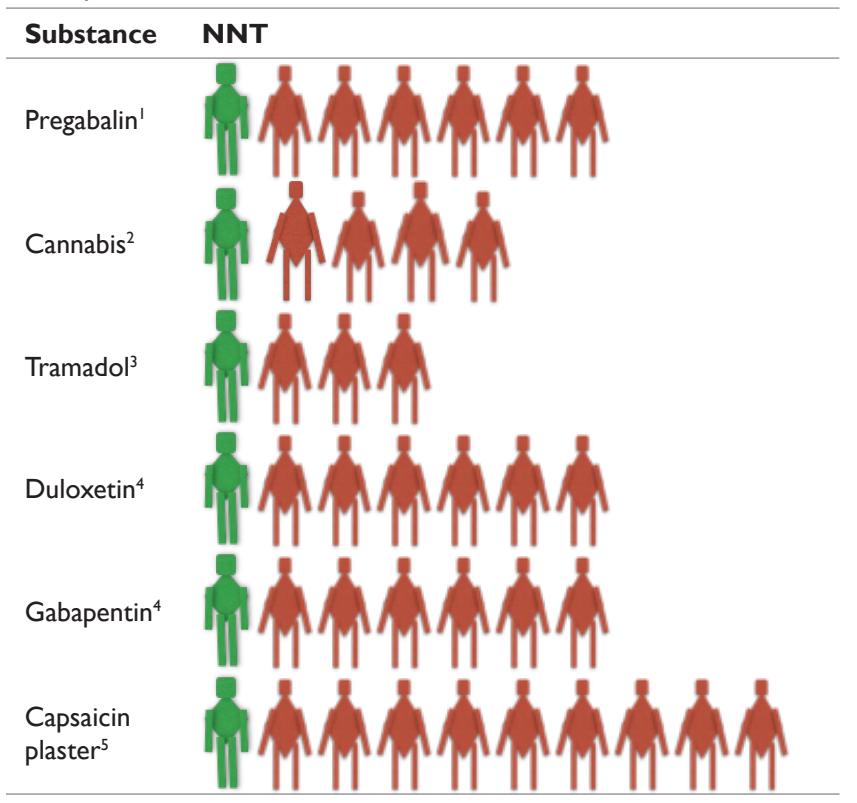

\section{A single-blind $\mathbf{N}$-of-I test paradigm, as a base for precision pain medicine}

There are indications that in a number of peripheral neuropathic pain disorders, the pathogenesis - at least for a part - is localized within the skin. Small lipophilic co-analgesics are therefore ideally suited for repositioning within peripheral neuropathic pain syndromes. Since most patients indicate that the onset of analgesic action is within 30 minutes after application of a cream containing a co-analgesic, we have developed a placebo-controlled response test for use in the clinical practice. We believe that an individualized pain treatment is possible in this way, because patients can indicate for themselves which cream benefits them most. Schork pointed out that a key component will be transforming everyday clinical care into solid N-of-1 trials. He also felt that: "key to making precision medicine mainstream is the 
ongoing shift in the relationship between patients and physicians. A major advantage of the N-of-1 approach over classical trials is that patients are no longer guinea pigs, whose involvement in a study may help only future generations. In $\mathrm{N}$-of-1 trials, the effectiveness of different treatments are vetted for the actual participants." (p.610)

It is exactly this we explored during the last years, based on our topical formulation containing phenytoin $10 \%$. For the purpose of the precision medicine we developed a single-blind placebo-controlled response test (SIBRET), see Figure 1. For the use of SIBRET a painful polyneuropathy is very well suited, because symptoms are mostly symmetrical (e.g. both feet). Examples of such polyneuropathy are PDN, CIAP and CIPN. Mostly the pain intensity is the same in both areas, and is measured on the 11-point numerical rating scale (NRS). On one foot the active cream will be applied and on the other foot placebo cream. The patient only knows that 2 different creams are applied on both feet. Responders are patients who experience 1) within 30 minutes, 2) a pain reduction of at least 2 point on the NRS in favor of the active cream. Responders will be prescribed the active cream. Non-responders can do another SIBRET with another topical analgesic. This way, no treatment delay will arise, and is in line with precision pain medicine. ${ }^{9}$

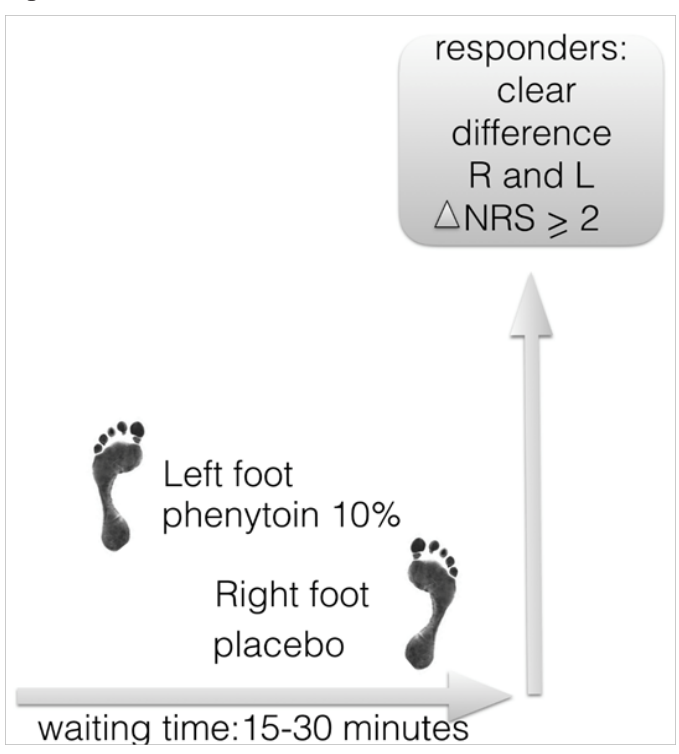

Figure I Single-blind response test paradigm: patients suffering from symmetrical painful neuropathy can be tested within a short period of 30 minutes, whether they are responders for phenytoin 10\% cream, or placebo. The same test can also be conducted in a double-blind version.

\section{Acknowledgements}

None.

\section{Conflict of interest}

Two authors (JMKH, DJK) are patent holders of two patents related to the topical formulations of phenytoin in the treatment of pain: 1) Topical phenytoin for the use in the treatment of peripheral neuropathic pain and 2) Topical pharmaceutical composition containing phenytoin and a (co-) analgesic to treat pain.

\section{References}

1. Finnerup NB, Haroutounian S, Baron R, et al. Neuropathic pain clinical trials: factors associated with decreases in estimated drug efficacy. Pain. 2018;159(11):2339-2346.

2. Lee G, Grovey B, Furnish T, et al. Medical Cannabis for Neuropathic Pain. Curr Pain Headache Rep. 2018;22(1):8.

3. Duehmke RM, Derry S, Wiffen PJ, et al. Tramadol for neuropathic pain in adults. Cochrane Database Syst Rev. 2017;6:CD003726.

4. Wiffen PJ, Derry S, Bell RF, et al. Gabapentin for chronic neuropathic pain in adults. Cochrane Database Syst Rev. 2017;6:CD007938.

5. Derry S, Wiffen PJ, Kalso EA, et al. Topical analgesics for acute and chronic pain in adults - an overview of Cochrane Reviews. Cochrane Database Syst Rev. 2017;5:CD008609.

6. Peng WW, Guo XL, Jin QQ, et al. Biological mechanism of post-herpetic neuralgia: Evidence from multiple patho-psychophysiological measures. Eur J Pain. 2017;21(5):827-842.

7. Kopsky DJ, Keppel Hesselink JM. Phenytoin Cream for the Treatment of Neuropathic Pain: Case Series. Pharmaceuticals (Basel). 2018;11(2):E53.

8. Schork NJ. Personalized medicine: Time for one-person trials. Nature. 2015;520(7549):609-611.

9. Mackey S. Future Directions for Pain Management: Lessons from the Institute of Medicine Pain Report and the National Pain Strategy. Hand Clin. 2016;32(1):91-98. 